KOBE-FHD-02-06

YNU-HEPTH-02-105

hep-ph/0212366

\title{
Charmed Hadron Production in Polarized $p p$ Reactions as a Probe of Polarized Gluons in the Proton
}

\author{
TOSHIYUKI MORII \\ Division of Sciences for Natural Environment, \\ Faculty of Human Development, \\ Kobe University, Nada, Kobe 657-8501, JAPAN \\ Electronic address:morii@kobe-u.ac.jp \\ KazUMASA OHKUMA \\ Department of Physics, \\ Faculty of Engineering, \\ Yokohama National University, \\ Hodogaya, Yokohama 240-8501, JAPAN \\ Electronic address:ohkuma@phys.ynu.ac.jp
}

\begin{abstract}
To probe the behavior of polarized gluons in the proton, we propose the charmed hadron, such as $\Lambda_{c}^{+}$and $D^{*}$, production in the forthcoming RHIC experiments. We found that the spin correlation between the target proton and the produced $\Lambda_{c}^{+}$ baryon might be a good signal for testing models of the polarized gluon distribution in the proton.
\end{abstract}

\footnotetext{
$\dagger$ Talk presented by K. Ohkuma at the XVI Particle and Nuclei International conference, Osaka, Sep. 30 - Oct. 4, 2002
} 


\section{INTRODUCTION}

The advent of so-called "the proton spin puzzle" which has emerged from the measurement of the polarized structure function of proton $g_{1}^{p}(x)$ by the EMC collaboration [1], has stimulated a great theoretical and experimental activities in nuclear and particle physics [2]. Though a great deal of efforts have been made for solving this puzzle so far, many problems still remain to be solved. As is well known, the spin of proton is carried by quarks, gluons and their orbital angular momenta. From the next-to-leading order QCD analysis for many and precise data on the polarized structure function $g_{1}(x)$ of nucleons, now we have a rather good knowledge on the polarized quark distribution in the proton. However, the polarized gluon distribution $\Delta G(x)$ in the nucleon is still very uncertain. To know how the gluon polarizes in the nucleon is very important to solve the proton spin puzzle. So far, the behavior of gluons in the proton have been studied in many cases for deep inelastic polarized $e-p$ scattering. However, the Relativistic Heavy Ion Collider(RHIC) could open another chance to probe internal structure of proton via $\vec{p} \vec{p}$ collisions. In the RHIC experiments [3], several interesting processes, such as high $p_{T}$ prompt photon production, jet production, heavy flavor production, etc. are proposed to probe the polarized gluon in the proton. Here we also propose another processes, i.e. the polarized charmed hadron production, $p \vec{p} \rightarrow \vec{\Lambda}_{c}^{+} X$ and $p \vec{p} \rightarrow \vec{D}^{*} X$, in the polarized proton-unpolarized proton collision to extract information about $\Delta G(x)$. $m$ In these processes, $\Lambda_{c}^{+}$is mainly produced via fragmentation of a charm quark which is originated dominantly from gluon-gluon fusion subprocess. Thus, its cross section is expected to be sensitive to the gluon distribution in the target proton. Moreover, since $\Lambda_{c}^{+}$is composed of a heavy charm quark and antisymmetrically combined light up and down quarks, the spin of $\Lambda_{c}^{+}$is basically carried by a charm quark which is produced via gluon-gluon fusion subprocess. Therefore, observation of the spin of the produced $\Lambda_{c}^{+}$gives us information about $\Delta G(x)$ in the nucleon.

\footnotetext{
${ }^{\sharp 1}$ In this report we focus only on the $\Lambda_{c}^{+}$production, though we have calculated for $D^{*}$ production, too, because the main point of the result remain unchanged.

$\sharp 2$ This is because charm quarks are tiny contents in the proton.
} 


\section{SPIN CORRELATION ASYMMETRY AND ITS STATISTICAL SENSITIVITY}

As a useful observable to extract $\Delta G(x)$ in the proton, we introduce a spin correlation asymmetry between the target polarized-proton and produced $\Lambda_{c}^{+}$baryon;

$$
A_{L L}=\frac{d \sigma_{++}-d \sigma_{+-}+d \sigma_{--}-d \sigma_{-+}}{d \sigma_{++}+d \sigma_{+-}+d \sigma_{--}+d \sigma_{-+}} \equiv \frac{d \Delta \sigma / d X}{d \sigma / d X}, \quad\left(X=p_{T} \text { or } \eta\right)
$$

where $d \sigma_{+-}$, for example, denotes the spin-dependent differential cross section with the positive helicity of the target proton and the negative helicity of the produced $\Lambda_{c}^{+}$baryon. $p_{T}$ and $\eta$, which are represented as $X$ in Eq.(1), are transverse momentum and pseudo-rapidity of produced $\Lambda_{c}^{+}$, respectively. The spin-independent(dependent) differential cross section $d(\Delta) \sigma / d X$ can be calculated by the quarkparton model (see ref. [4] for details).

Statistical sensitivities of $A_{L L}$ for the $p_{T}$ and $\eta$ distribution are estimated by using the following formula;

$$
\delta A_{L L} \simeq \frac{1}{P} \frac{1}{\sqrt{b_{\Lambda_{c}^{+}} \epsilon L T \sigma}} .
$$

To numerically estimate the value of $\delta A_{L L}$, here we use following parameters: operating time; $T=100$-day, the beam polarization; $P=70 \%$, a luminosity; $L=$ $8 \times 10^{31}\left(2 \times 10^{32}\right) \mathrm{cm}^{-2} \mathrm{sec}^{-1}$ for $\sqrt{s}=200(500) \mathrm{GeV}$, the trigger efficiency; $\epsilon=10 \%$ for detecting produced $\Lambda_{c}^{+}$events and a branching ratio; $b_{\Lambda_{c}^{+}} \equiv \operatorname{Br}\left(\Lambda_{c}^{+} \rightarrow\right.$ $\left.p K^{-} \pi^{+}\right) \simeq 5 \%$ [5]. The branching ratio of this purely charged decay mode is needed to measure the polarization of produced $\Lambda_{c}^{+} . \sigma$ denotes the unpolarized cross section integrated over suitable $p_{T}$ or $\eta$ region.

\section{NUMERICAL ANALYSIS}

In the numerical calculation of $A_{L L}$, we limited the integration region of $\eta$ and $p_{T}$ of produced $\Lambda_{c}^{+}$as $-1.3 \leq \eta \leq 1.3$ and $3 \mathrm{GeV} \leq p_{T} \leq 15(40) \mathrm{GeV}$, respectively, for $\sqrt{s}=200(500) \mathrm{GeV}$. The range of $\eta$ and the lower limit of $p_{T}$ were selected in order to get rid of the contribution from the diffractive $\Lambda_{c}^{+}$production. As for the upper limit of $p_{T}$, we took it as described above, for simplicity, though the kinematical maximum of $p_{T}$ of produced $\Lambda_{c}^{+}$is slightly larger than $15 \mathrm{GeV}$ and $40 \mathrm{Gev}$ for $\sqrt{s}=200 \mathrm{GeV}$ and $500 \mathrm{GeV}$, respectively. In addition, we took the 
AAC [6] and GRSV01 [7] parameterization models for the polarized gluon distribution function and the GRV98 [8] model for the unpolarized one. Though both of AAC and GRSV01 models excellently reproduce the experimental data on the polarized structure function of nucleons $g_{1}(x), \Delta G(x)$ for those models are quite different. Therefore, those models should be tested in other processes. Since our process is semi-inclusive, the fragmentation function of a charm quark to $\Lambda_{c}^{+}$is necessary to carry out numerical calculations. For the unpolarized fragmentation function, we used Peterson fragmentation function, $D_{c \rightarrow \Lambda_{c}^{+}}(z)$ [5, 9]. However, since we have no data, at present, about polarized fragmentation functions $\Delta D_{\vec{c} \rightarrow \vec{\Lambda}_{c}^{+}}(x)$ for the polarized $\Lambda_{c}^{+}$production, we took the following ansatz for it

$$
\Delta D_{\vec{c} \rightarrow \vec{\Lambda}_{c}^{+}}(z)=C_{c \rightarrow \Lambda_{c}^{+}} D_{c \rightarrow \Lambda_{c}^{+}}(z),
$$

where $C_{c \rightarrow \Lambda_{c}^{+}}$is a scale-independent spin transfer coefficient. In this analysis, we studied two cases: (A) $C_{c \rightarrow \Lambda_{c}^{+}}=1$ (non-relativistic quark model) and (B) $C_{c \rightarrow \Lambda_{c}^{+}}=$ $z$ (Jet fragmentation model [10]). As we discussed before, if the spin of $\Lambda_{c}^{+}$is equal to the spin of the charm quark produced in the subprocess, the model (A) might be a reasonable scenario.

\section{RESULTS AND DISCUSSION}

Numerical results of $A_{L L}$ are shown in Fig. 1 and Fig. 2. In these figures, we attached $\delta A_{L L}$ to the solid line of $A_{L L}$ calculated for the case of the GRSV01 parametrization model of polarized gluon and the non-relativistic fragmentation model. H3 $^{3}$ Comparing with those figures, we can see that the $\eta$ distributions of $A_{L L}$ are more effective than the $p_{T}$ distributions at $\sqrt{s}=200 \mathrm{GeV}$ and $500 \mathrm{GeV}$ to distinguish various models. As shown in the right panel of Fig. 2 given at $\sqrt{s}=500 \mathrm{GeV}$, we could distinguish the parametrization models of polarized gluon as well as the models of the spin-dependent fragmentation function, though the magnitude of $A_{L L}$ is rather small. At $\sqrt{s}=200 \mathrm{GeV}$, the magnitude of $A_{L L}$ for $\eta$ distribution becomes larger, though statistical sensitivities are not so small. If the integrated luminosity at $\sqrt{s}=200 \mathrm{GeV}$ becomes large and the detection efficiency $\epsilon$ is improved, this observable could be promising to distinguish not only the models of $\Delta G(x)$ but also the models of $\Delta D(z)$. On the other hand, we cannot say anything

\footnotetext{
$\sharp 3$ Note that as shown from Eq.(2), $\delta A_{L L}$ does not depend on both of the model of polarized gluons and the model of fragmentation functions.
} 

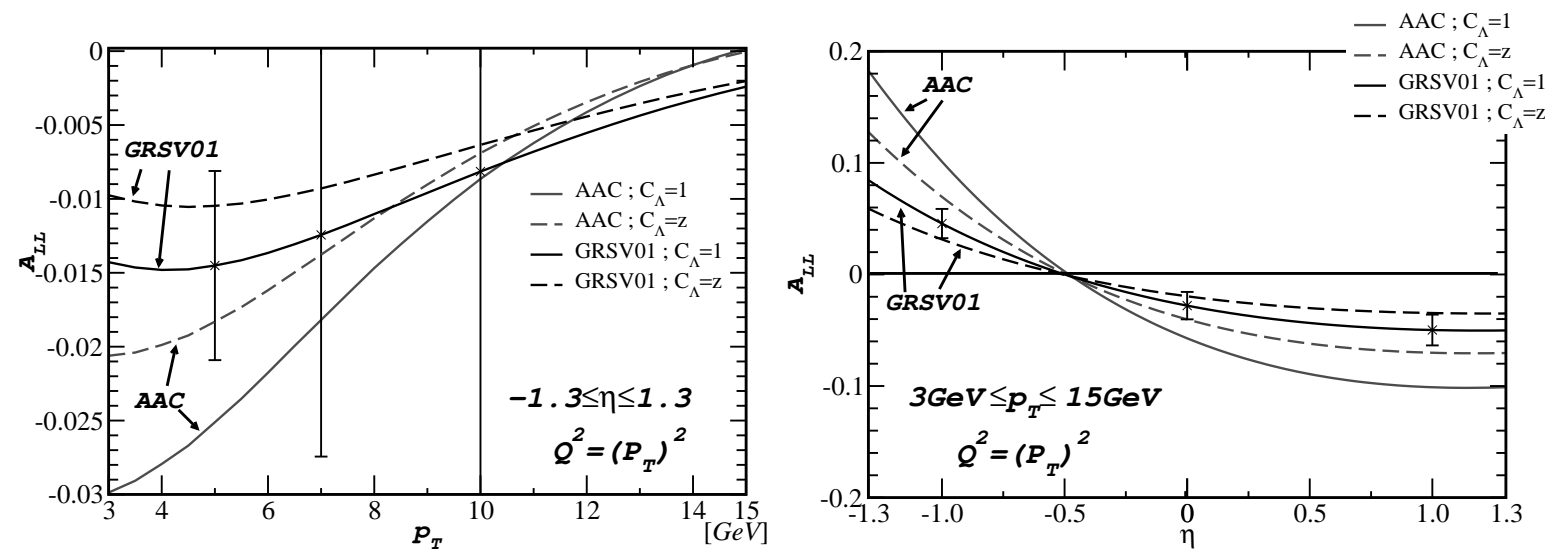

Figure 1: Spin correlation asymmetry of $p_{T}$ (left panel) and $\eta$ (right panel) distribution at $\sqrt{s}=200 \mathrm{GeV}$
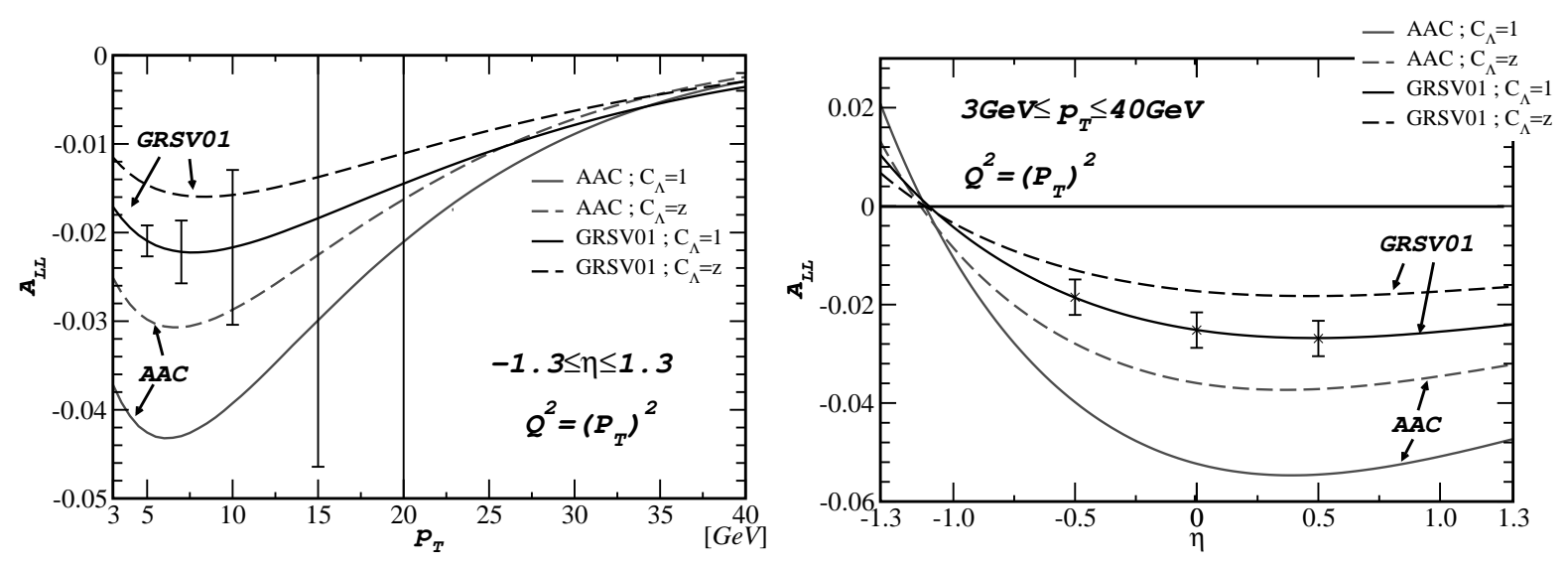

Figure 2: The same as in Fig. 1, but for $\sqrt{s}=500 \mathrm{GeV}$

from the $p_{T}$ distribution of $A_{L L}$ at high $p_{T}$ region, since $\delta A_{L L}$ becomes rapidly large with increasing $p_{T}$. However, if we confine the kinematical region in rather small $p_{T}$ range such as $p_{T}=3 \sim 5(10) \mathrm{GeV}$ at $\sqrt{s}=200(500) \mathrm{GeV}$, it might be still effective.

Though this analysis is confined to leading order, the results are interesting and we hope our prediction will be tested in the forthcoming RHIC experiment. 


\section{REFERENCES}

[1] J. Ashman et al., EMC Collab., Phys. Lett. B206 1988 364; J. Ashman et al., EMC Collab., Nucl. Phys. B328 19891.

[2] For a review see: H. Y. Cheng, Int. J. Mod. Phys. A11 (1996) 5109; B. Lampe and E. Reya, Phys. Rep. 332 (2000) 1; H. Y. Cheng, Chin. J. Phys. 38 (2000) 753, hep-ph/0002157; B. W. Filippone and X. Ji, hep-ph/0101224.

[3] G. Bunce, N. Saito, J. Soffer and W. Vogelsang, Ann. Rev. Nucl. Part. Sci. 50 (2000) 525 .

[4] K. Ohkuma, K. Sudoh and T. Morii, Phys. Lett. B491 (2000) 117, [Erratumibid. B543 (2002) 323]; K. Ohkuma, T. Morii and S. Oyama, hep-ph/0201144; T. Morii and K. Ohkuma, hep-ph/0211046.

[5] K. Hagiwara et al, Phys. Rev. D66 (2002) 010001.

[6] Y. Goto et al. [Asymmetry Analysis Collaboration], Phys. Rev. D62(2001) 034017.

[7] M Glück, E. Reya, M. Stratmann and W. Vogelsang, Phys. Rev. D 63 (2001) 094005.

[8] M. Glück, E. Reya and A. Vogt, Eur. Phys. J. C5 (1998) 461.

[9] C. Peterson, D. Schlatter, I. Schmitt and P. M. Zerwas, Phys. Rev. D27 (1983) 105.

[10] A. Bartl, H. Fraas and W. Majerotto, Z. Phys. C6 (1980) 335. 